\title{
Reference-Dependent Preferences, Super-Dominance and Stochastic Stability
}

\author{
Ryoji Sawa and Jiabin Wu*
}

August 8, 2018

\begin{abstract}
This paper investigates stochastic stability of noisy best response dynamics with referencedependent preferences. We define a strategy as super-dominant in a $2 \times 2$ coordination game if it is risk-dominant and the maximin strategy in terms of monetary returns and all players playing it constitutes an equilibrium which Pareto-dominates all other equilibria. If such a strategy exists, the corresponding equilibrium, which we call the super-dominant equilibrium, is uniquely stochastically stable for the BRM choice rule (the best response choice rule with uniform random errors) given any model of reference-dependent preferences. However, for any $2 \times 2$ coordination game with a super-dominant strategy, there exists a model of reference-dependent preferences with which the super-dominant equilibrium fails to be stochastically stable for the logit choice rule.
\end{abstract}

Keywords: Evolutionary game theory; Behavioral game theory; Equilibrium selection; Stochastic Stability; Reference-dependent preferences; Loss-aversion; Loss-avoidance; Maximin; Risk-dominance; Payoff-dominance.

JEL Code: C72, C73.

*Sawa: Department of Policy and Planning Sciences, University of Tsukuba, email: rsawa@sk.tsukuba.ac.jp. Wu: Department of Economics, University of Oregon, email: jwu5@uoregon.edu. Please direct correspondence to either author. 


\section{Introduction}

A growing body of experimental evidence suggests that people's preferences are reference-dependent. That is, outcomes are evaluated in relative terms rather than in absolute terms. ${ }^{1}$ Yet, little is known about how and to what extent reference-dependent preferences affect the long-run behavior of individuals in a society. This paper attempts to answer this question by employing stochastic stability analysis from evolutionary game theory.

Stochastic stability analysis (Foster and Young, 1990) offers a framework to study dynamic processes, and deduces predictions of social conventions selected by a population of individuals in the long run. It is known that, when individuals obey expected utility theory for non-forward looking behavior within a single period, stochastic stability analysis uniquely selects the risk-dominant equilibrium in $2 \times 2$ coordination games if individuals adopt the best response with mutations rule (BRM) (Kandori et al. 1993 and Young 1993), i.e. the best response choice rule (Cournot, 1838) plus uniform errors, or the logit choice rule (Blume 1993). ${ }^{2}$ Since then, a variety of choice rules have been examined in different contexts. For example, among many others, the BRM rule is examined by Ellison (2000) and Neary (2012), the logit choice rule is examined by Blume (2003), Hwang and Newton (2017) and Hwang and Rey-Bellet (2017), and the probit choice rule is examined by Myatt and Wallace (2003) and Dokumacı and Sandholm (2011). There are also many studies that examine some classes of noisy best response choice rules, e.g. Bergin and Lipman (1996), Maruta (2002), Naidu et al. (2010), Peski (2010), Sandholm (2010a), Staudigl (2012), Kreindler and Young (2013), Staudigl and Weidenholzer (2014), Newton and Sawa (2015), Bilancini and Boncinelli (2016), Klaus and Newton (2016), Sandholm and Staudigl (2016), and Newton (2018a). For experimental investigations, see Lim and Neary (2016), Mäs and Nax (2016), and Hwang et al. (2018). See also Newton (2018b) for a survey of recent works.

Recently, Sawa and Wu (2018) studied stochastically stable states of games with players who have a specific type of reference-dependent preferences, loss-aversion proposed by Kahneman and Tversky (1979), which is restricted to individual periods. That is, agents' perceived losses are weighed more heavily than their perceived gains in each single period. ${ }^{3}$ They found that stochastic stability of the risk-dominant equilibrium is no longer guaranteed given that agents are loss-averse

\footnotetext{
${ }^{1}$ See for example, Kahneman and Tversky (1979) and Köszegi and Rabin (2006).

${ }^{2}$ The terminology "the best response with mutations rule (BRM)" follows extant studies, e.g. Sandholm (2010b), and it refers to a stochastic choice rule under which every suboptimal strategy is equally likely to be chosen.

${ }^{3}$ See also Thaler (1980), Knetsch and Sinden (1984), Tversky and Kahneman (1992a) and Köszegi and Rabin (2006).
} 
and they show that an equilibrium is always stochastically stable under the BRM choice rule if the strategy played in the equilibrium is both risk-dominant and a maximin strategy in terms of monetary returns.

In this paper, we extend the analysis to a more general class of reference-dependent preferences restricted to individual periods: First, we allow agents' reference points to take various forms. A reference point can be an agent's status-quo, an expected outcome, an outcome of some foregone action, or any outcome an agent could think that "might have been". ${ }^{4}$ As Sawa and Wu (2018) pointed out, stability of equilibria may be affected by reference points that players have. Second, the mechanism of evaluating outcomes relative to reference points may also vary. People may exhibit loss aversion. Alternatively, people may exhibit loss avoidance as proposed in Cachon and Camerer (1996). That is, people do not choose actions that result in certain losses. ${ }^{5}$ We find that, given individuals in the population have reference-dependent preferences, a strong concept, which we call super-dominance, serves as an important condition for stochastic stability.

We define a strategy in a $2 \times 2$ coordination game to be super-dominant if it satisfies two conditions in terms of monetary returns: (i) it is a (weak) maximin strategy, and (ii) the equilibrium where all players play that strategy is weakly payoff-dominant, with at least one of the two conditions being strict. We call a strategy profile a super-dominant equilibrium if all individuals play a super-dominant strategy. Note that with at least one of the two conditions being strict, superdominance directly implies strict risk-dominance. Both properties have been shown to give an individual intuitive reasons for choosing a strategy. ${ }^{6}$ Hence, a super-dominant strategy (if exists) is arguably the most salient strategy for individuals to coordinate on. Note that, Proposition 4.1 of Maruta (2002) shows that super-dominance is a sufficient condition for stochastic stability for a broad class of payoff-dependent mistake models.

We first consider the BRM choice rule and find that an equilibrium is uniquely stochastically stable under the BRM rule for all models of reference-dependent preferences if and only if it is superdominant. Risk-dominance (in terms of monetary outcomes) is sufficient to guarantee stochastic stability in $2 \times 2$ coordination games if utility functions are linear in monetary outcomes. We show that for a broader class of utility functions, not only risk-dominance but also payoff-dominance and

\footnotetext{
${ }^{4}$ Chapter 26 of Kahneman (2011) offers a brief discussion on difficulties of choosing appropriate reference points and the dependence of reference points on situations.

${ }^{5}$ See also Rydval and Ortmann (2005), Feltovich (2011) and Feltovich et al. (2012) for experimental evidence on loss-avoidance.

${ }^{6}$ See von Neumann and Morgenstern (1944) for a maximin strategy, and Harsanyi and Selten (1988) for riskdominance and payoff-dominance.
} 
being a maximin strategy are required to guarantee stochastic stability under the BRM rule.

Second, we find that for the logit choice rule, super-dominance does not guarantee stochastic stability. For any $2 \times 2$ game with a super-dominant equilibrium, there exists a model of referencedependent preferences with which it fails to be stochastically stable under the logit rule. Nevertheless, if agents adopt some constant reference point, we show that super-dominance still serves as a tight condition for stochastic stability.

Although super-dominance is a rather strong concept, it is not unusual to consider such a concept and investigate its influence on long-run social conventions. For example, games with a super-dominant strategy have been examined in the literature on dynamic technology diffusion. In a typical model in this literature, there are two technologies, a status-quo and a new technology. The new technology is often assumed to be super-dominant. Hence, it is superior comparing to the status-quo. Morris (2000), Young (2011), Kreindler and Young (2014), and Newton and Angus (2015), among many others, study whether a population in which all individuals initially adopt the status-quo technology will eventually switch to the new technology through dynamic processes and how long such a transition would take place. ${ }^{7}$ Our results suggest that whether the new technology can become prevalent in a society with reference-dependent individuals depend on the choice rules adopted by the individuals.

The paper is organized as follows. Section 2 provides the model. Section 3 conducts analysis on stochastic stability. Section 4 discusses the tightness of the conditions on reference-dependent preferences. Section 5 concludes.

\section{The Model}

\subsection{The Population Game and Its Dynamics}

We consider a discrete-time dynamic process in which $n<\infty$ identical agents are repeatedly and randomly matched to play a coordination game. Let $N=\{1, \ldots, n\}$ denote the set of agents. We restrict our attention to $2 \times 2$ symmetric coordination games with strategy space $S=\{0,1\}$. The monetary returns received from the coordination game are illustrated in Table 1 . The state of the process is given by the fraction of agents playing strategy 1 . Let $X \equiv\left\{0, \frac{1}{n}, \frac{2}{n}, \ldots, 1\right\}$ denote the state space and $x \in X$ denote the current state. We assume that $x$ is observed by every agent. For $s \in S$, the state in which all agents play strategy $s$ is called all-s.

\footnotetext{
${ }^{7}$ The focus of those studies is not on super-dominant strategies. Some of the studies restrict their attention to
} 
Table 1: $2 \times 2$ Coordination Game (Monetary Returns)

Player 2

\begin{tabular}{cc|c|c|}
\multicolumn{1}{c}{0} & \multicolumn{1}{c}{0} \\
\cline { 3 - 4 } Player 1 & 0 & $a_{00}, a_{00}$ & $a_{01}, a_{10}$ \\
\cline { 3 - 4 } & 1 & $a_{10}, a_{01}$ & $a_{11}, a_{11}$ \\
\cline { 3 - 4 } & & &
\end{tabular}

The monetary returns from the game satisfy $a_{00}, a_{01}, a_{10}, a_{11}>0, a_{00}>a_{10}$ and $a_{11}>a_{01}$. We will shortly assume that agents' utility functions are strictly increasing in monetary returns, which implies that $(0,0)$ and $(1,1)$ are strict Nash equilibria.

Each agent occasionally reconsiders her choice of strategy, using some myopic best response rule. Myopic best responses for an agent are not determined by monetary returns but by her perceived utilities, which will be introduced in Section 2.2. Each agent's myopic best response rule is assumed to be perturbed by a small stochastic noise, which allows us to select an equilibrium based on the robustness of the equilibrium against the noise. The choice rule, the dynamics and the selection concept will be introduced more precisely in Section 2.3 .

\subsection{Reference-dependent preferences}

Agents' preferences are reference-dependent. That is, each agent has a reference point which determines whether she perceives the monetary return from the game as a gain or a loss. The reference point of each agent $i \in N$ is given by a function $r_{i}:[0,1] \rightarrow \mathbb{R}$, which maps each population state to her reference point. In other words, we allow an agent's reference point to be affected by other agents' behavior.

For simplicity, we assume that all agents have the same reference point in the major part of the paper. However, our main results, Theorems 3.4 and 3.5, hold for agents who have heterogeneous reference points.

An agent $i \in N$ with reference-dependent preferences who anticipates receiving a monetary payoff $a$ will assign a value to it, which is denoted by $v\left(a, r_{i}(x)\right)$. We assume that the value function $v(\cdot, \cdot): \mathbb{R} \times \mathbb{R} \rightarrow \mathbb{R}$ satisfies some or all of the following general conditions for each of our results.

games with a super-dominant strategy for simplicity's sake. 
Assumption 2.1 (value function).

$$
\begin{array}{lll}
\text { (i) } & v(a, r)>0 & \text { if } a>r, \\
(\text { ii }) & v(a, r)=0 & \text { if } a=r, \\
(\text { iii }) & v(a, r)<0 & \text { if } a<r, \\
(\text { iv }) & v(a, r)>v\left(a^{\prime}, r\right) & \forall a>a^{\prime}, \\
(v) & v((1+\gamma) r, r) \leq-v((1-\gamma) r, r) & \forall \gamma>0 .
\end{array}
$$

The first three conditions indicate that the agents derive positive values from gains and negative values from losses. The fourth condition indicates that a greater monetary return is always strictly better than a smaller one. For the fifth condition, observe that $(1+\gamma) r-r=\gamma r=|(1-\gamma) r-r|$. So the condition captures a well-known phenomenon that losses are weighed more heavily than equalsized gains (Kahneman and Tversky (1979)). The five conditions are satisfied by many known value functions capturing reference-dependent preferences. Below, we provide two examples.

Example 1 (loss-aversion). Tversky and Kahneman (1992b) propose the following value function to capture loss-averse preferences.

$$
v(a, r)= \begin{cases}(a-r)^{\alpha} & \text { if } a-r \geq 0 \\ -\lambda(r-a)^{\beta} & \text { otherwise }\end{cases}
$$

for $\beta \geq \alpha>0$ and $\lambda \geq 1$. Parameters $\alpha$ and $\beta$ measure the diminishing sensitivity of an agent's valuations on gains and losses, respectively. Also, losses are weighed more heavily than gains proportionally by $\lambda$.

Example 2 (loss-avoidance). Feltovich et al. (2012) propose the following value function to capture loss-avoidance preferences:

$$
v(a, r)= \begin{cases}(a-r) & \text { if } a-r \geq 0 \\ (a-r)-B & \text { otherwise }\end{cases}
$$

for $B>0$. The value function indicates that losses are weighed more heavily than gains by some lump-sum disutility $B$.

Given the value function, the utility of an agent $i \in N$ of choosing strategy $s \in\{0,1\}$ is given by

$$
u_{s}\left(x, r_{i}(x)\right)=(1-x) v\left(a_{s 0}, r_{i}(x)\right)+x v\left(a_{s 1}, r_{i}(x)\right)
$$


which is the expectation of her value functions. The probability weights are objective and equal to the probabilities that this agent meets agents with different strategies. ${ }^{8}$

\subsection{Prospect Dynamic and Stochastically Stable States}

The agents are recurrently matched to play the coordination game in Table 1. In each period $t \in\{1,2, \ldots\}$, one agent is randomly chosen and given an opportunity to revise her strategy. Given the revision opportunity, an agent $i \in N$ follows the noisy best response revision protocol:

$$
\begin{aligned}
& \rho_{01}\left(x, r_{i}(x)\right)=\sigma^{\eta}\left(u_{1}\left(x, r_{i}(x)\right)-u_{0}\left(x, r_{i}(x)\right)\right), \\
& \rho_{10}\left(x, r_{i}(x)\right)=\sigma^{\eta}\left(u_{0}\left(x, r_{i}(x)\right)-u_{1}\left(x, r_{i}(x)\right)\right),
\end{aligned}
$$

where $\sigma^{\eta}: \mathbb{R} \rightarrow[0,1]$ and $\eta>0$ denotes the noise (error) level. When agent $i$ receives an opportunity to revise her strategy, she switches from strategy 0 to 1 with probability $\rho_{01}\left(x, r_{i}(x)\right)$ if she is currently using strategy 0 ; and she switches from strategy 1 to 0 with probability $\rho_{10}\left(x, r_{i}(x)\right)$ if she is currently using strategy 1 . We assume that $\sigma^{\eta}(\cdot)$ becomes the best response protocol when the noise level approaches zero, that is,

$$
\lim _{\eta \rightarrow 0} \sigma^{\eta}(a)= \begin{cases}1 & \text { if } a>0, \\ 0 & \text { if } a<0 .\end{cases}
$$

We call the dynamic generated by (3) and (4) the prospect dynamic. In particular, we adopt two widely used revision protocols, the best response with mutations (BRM) choice rule from Kandori et al. (1993) and the logit choice rule from Blume (1993).

Example 3 (BRM choice rule). The choice probabilities are given by

$$
\sigma^{\eta}(a)= \begin{cases}1-\exp \left(-\eta^{-1}\right) & \text { if } a>0, \\ \exp \left(-\eta^{-1}\right) & \text { if } a<0\end{cases}
$$

where $\exp \left(-\eta^{-1}\right)$ can be considered as the mutation rate, $\varepsilon$.

Example 4 (logit choice rule). The choice probabilities with noise level $\eta>0$ are given by

$$
\sigma^{\eta}(a)=\frac{\exp \left(\eta^{-1} a\right)}{1+\exp \left(\eta^{-1} a\right)} .
$$

\footnotetext{
${ }^{8}$ The probability weights people place on different lottery outcomes may be subjective and potentially be influenced by loss-aversion (see Tversky and Kahneman (1992b)). For simplicity, we do not consider such a possibility in this paper.
} 
The logit choice rule can be derived from a random utility model in which the utility for each strategy is perturbed by i.i.d. random variables with the Gumbel distribution, which is similar to a normal distribution. The main difference between the two choice rules is in the exponential decay rates of error probabilities. The exponential decay rate for the BRM rule is uniform for all errors while that for the logit choice rule depends on the amount of payoff decrease caused by each error.

The model described above defines a Markov chain $\left\{X_{t}^{n}\right\}$ on the discrete state space $X$. We focus on the stationary distribution $\mu$ of the Markov chain when the noise level approaches zero and population size approaches infinity. A state is said to be stochastically stable if it has a non-negligible weight in the limiting distribution.

Definition 2.2 (stochastic stability). A state $x \in X$ is stochastically stable if $\lim _{\eta \rightarrow 0} \mu(x)>0$ for all sufficiently large $n$.

Here we provide some intuitions for how to determine stochastically stable states under the two choice rules. Recall that in Section 2.2, for simplicity, we assume that all agents have the same reference point, i.e. $r_{i}(x)=r(x)$ for some $r(\cdot):[0,1] \rightarrow \mathbb{R}$ and all $i \in N$. Define

$$
\begin{aligned}
& \kappa_{+}(x)=-\lim _{\eta \rightarrow 0} \eta \log \left[\sigma^{\eta}\left(u_{1}(x, r(x))-u_{0}(x, r(x))\right)\right], \\
& \kappa_{-}(x)=-\lim _{\eta \rightarrow 0} \eta \log \left[\sigma^{\eta}\left(u_{0}(x, r(x))-u_{1}(x, r(x))\right)\right] .
\end{aligned}
$$

$\kappa_{+}(x)$ and $\kappa_{-}(x)$ are the exponential decay rates of the transition probability from $x$ to $x+\frac{1}{n}$ and from $x$ to $x-\frac{1}{n}$, respectively. For example, Eq.(6) and (7) imply that if strategy 0 is the unique best response in state $x$, then $\kappa_{+}(x)=1$ for the BRM rule and $\kappa_{+}(x)=u_{0}(x, r(x))-u_{1}(x, r(x))$ for the logit choice. The one dimensional state space together with that exactly one agent revises in each period implies that the process is a birth and death process. It is known (see Durrett 2005) that the stationary distribution of the process can be expressed as

$$
\frac{\mu(x)}{\mu(0)}=\prod_{y=1}^{n x} \frac{n-y+1}{y} \frac{\sigma^{\eta}\left(u_{1}\left(\frac{y-1}{n}, r\left(\frac{y-1}{n}\right)\right)-u_{0}\left(\frac{y-1}{n}, r\left(\frac{y-1}{n}\right)\right)\right)}{\sigma^{\eta}\left(u_{0}\left(\frac{y}{n}, r\left(\frac{y}{n}\right)\right)-u_{1}\left(\frac{y}{n}, r\left(\frac{y}{n}\right)\right)\right)} .
$$

Taking logarithms, multiplying by $\eta / n$, and taking the limit of the small noise level $\eta$ yields

$$
\lim _{\eta \rightarrow 0} \frac{\eta}{n} \log \frac{\mu(x)}{\mu(0)}=\frac{1}{n} \sum_{y=1}^{n x} \kappa_{-}\left(\frac{y}{n}\right)-\kappa_{+}\left(\frac{y-1}{n}\right) .
$$

Recall that all-s is the state in which all agents play strategy $s$. Given that all-0 and all-1 are candidates of stochastically stable states, if $\lim _{\eta \rightarrow 0} \frac{\eta}{n} \log \frac{\mu(1)}{\mu(0)}<0$, then all-0 is uniquely stochastically stable, and if the opposite inequality holds, then all-1 is uniquely stochastically stable. 
For the BRM choice rule, we employ the 'mutation counting' technique to characterize stochastically stable states. This technique is an implication of Freidlin and Wentzell (1998) and is shaped by Kandori et al. (1993), Young (1993) and their successors. Theorem 1 of Sandholm (2010a) shows that, for all sufficiently large $n$, this technique is reduced to examine which strategy has a greater basin of attraction on the continuous interval [0,1]. For example, all-0 is uniquely stochastically stable if there exists some $\gamma>1 / 2$ such that strategy 0 is the unique best response for all $x \in[0, \gamma]$.

For the logit choice rule, the exponential decay rate of the transition probability from $x$ to $x+\frac{1}{n}$ is $\kappa_{+}(x)=\left[u_{0}(x, r(x))-u_{1}(x, r(x))\right]_{+}$for all $x \in X .{ }^{9}$ Similarly, the exponential decay rate for the opposite move is $\kappa_{-}(x)=\left[u_{1}(x, r(x))-u_{0}(x, r(x))\right]_{+}$. Substituting those into Eq.(8), we find that all-0 state is uniquely stochastically stable if $\sum_{x \in X} u_{1}(x, r(x))-u_{0}(x, r(x))<0$.

It is known that if we choose an appropriate ordinal potential function for a given choice rule, stochastic stability in $2 \times 2$ symmetric games is characterized by potential maximization (see Blume 2003; Maruta 2002; Sandholm 2010a). We will discuss such potential functions corresponding to our settings and relate them to stochastically stable states in Section 4.2.

\section{Super-dominance and its stochastic stability}

\subsection{Super-Dominance}

We define a concept called super-dominance which combines three important concepts in game theory: risk-dominance, payoff-dominance and maximin strategy. ${ }^{10}$

Definition 3.1. Strategy $s \in\{0,1\}$ is a super-dominant strategy if the following two conditions are satisfied for $s^{\prime} \neq s$ and at least one is strict.

(1) $\min \left\{a_{s 0}, a_{s 1}\right\} \geq \min \left\{a_{s^{\prime} 0}, a_{s^{\prime} 1}\right\}$,

(2) $a_{s s} \geq a_{s^{\prime} s^{\prime}}$.

A population state in which every agent is playing the super-dominant strategy $s \in\{0,1\}$ (all-s) is a super-dominant equilibrium.

The first condition of Definition 3.1 states that it is the (weak) maximin strategy. Hence, this concept captures agents' tendency to avoid losses, particularly the loss occurred in the worst case

\footnotetext{
${ }^{9}[y]_{+}=y$ if $y>0$ and $[y]_{+}=0$ if $y \leq 0$.

${ }^{10}$ We define the condition in terms of (usually observable) monetary payoffs as in experimental studies Battalio et al. (2001) and Schmidt et al. (2003). Our intention is to examine if such a condition on monetary payoffs guarantee stochastic stability given that agents have reference-dependent preferences.
} 
scenario. The second condition states that the equilibrium corresponding to the super-dominant strategy Pareto-dominates the other equilibrium. A strategy $s$ is said to be risk-dominant if $a_{s 0}+$ $a_{s 1} \geq a_{s^{\prime} 0}+a_{s^{\prime} 1}$ for $s^{\prime} \neq s$. The two conditions with at least one condition being strict imply that a super-dominant strategy must be the unique risk-dominant strategy.

\subsection{The BRM choice rule and stochastic stability of super-dominance}

Sawa and $\mathrm{Wu}$ (2018) define a strategy to be loss-dominant if it is risk dominant and the (weak) maximin strategy. They show that a state in which all agents adopt the loss-dominant strategy is stochastically stable under the prospect dynamic with BRM choice rule, given that agents have loss-averse preferences with no diminishing sensitivity (i.e. $\alpha=\beta=1$ in Eq.(1)). In this paper, we consider a broader set of reference-dependent preferences. The following two lemmas demonstrate why loss-dominance is not sufficient for stochastic stability given that agents have reference-dependent preferences and why payoff-dominance is needed.

Lemma 3.2. Suppose all agents have loss-averse preferences as given in Eq.(1), If s is not payoffdominant, then there exist some $\alpha, \beta, \lambda$ and $\left\{r_{i}(x)\right\}_{i \in N}$ such that all-s state is not stochastically stable under the prospect dynamic with BRM choice rule.

Proof. Without loss of generality, let strategy 0 be a maximin strategy but not payoff dominant. We must have $a_{11}>\max \left\{a_{00}, a_{01}\right\}>a_{10}$. Let $r_{i}(x)=r$ for some constant $r \in\left(\max \left\{a_{00}, a_{01}\right\}, a_{11}\right)$ for all $x \in X$ and all $i \in N$.

Choose $\delta \in(0,1)$, let $\beta<1$ and choose $\beta$ such that

$$
\left(r-a_{10}\right)^{\beta}-\left(r-a_{00}\right)^{\beta}<\delta<\left(r-a_{01}\right)^{\beta}
$$

Note that the left-hand and right-hand sides approach 0 and 1, respectively, as $\beta$ approaches 0 . Hence, an $\beta$ satisfying the above inequalities exists. Observe that

$$
\begin{aligned}
u_{1}(x, r)-u_{0}(x, r) & =x\left(a_{11}-r\right)^{\alpha}-(1-x) \lambda\left(\left(r-a_{10}\right)^{\beta}-\left(r-a_{00}\right)^{\beta}\right)+x \lambda\left(r-a_{01}\right)^{\beta} \\
& >x\left(a_{11}-r\right)^{\alpha}+\lambda \delta(2 x-1) .
\end{aligned}
$$

For $x \in[1 / 2,1], x\left(a_{11}-r\right)^{\alpha}+\lambda \delta(2 x-1)$ is strictly positive. Since $u_{0}(x, r)$ and $u_{1}(x, r)$ are continuous on $x \in(0,1)$, all-1 is uniquely stochastically stable.

Note that when $\beta=1$, we have $u_{1}\left(\frac{1}{2}, r\right)-u_{0}\left(\frac{1}{2}, r\right)<0$ as long as 0 is loss-dominant defined in Sawa and $\mathrm{Wu}$ (2018), implying that the payoff-dominant equilibrium (all-1) is not stochastically 
stable. However, as $\beta$ decreases, agents start to experience less disutility in the loss frame and they become more risk-seeking. When agents' reference point $r \in\left(\max \left\{a_{00}, a_{01}\right\}, a_{11}\right)$, the only positive gain comes from $a_{11}$. Hence, when agents are sufficiently risk-seeking (low $\beta$ ), they are more willing to choose strategy 1 to achieve the positive gain. Lemma 3.2 highlights that allowing for diminishing sensitivity to losses $(\beta<1)$ can give rise to new results not accounted for in Sawa and $\mathrm{Wu}(2018) \cdot{ }^{11}$

Lemma 3.3. Suppose all agents have loss-avoidance preferences as given in Eq.(2). If $s$ is not payoff-dominant, then there exist some $B$ and $\left\{r_{i}(x)\right\}_{i \in N}$ such that all-s state is not stochastically stable under the prospect dynamic with BRM choice rule.

Proof. Without loss of generality, let strategy 0 be the one that is not payoff-dominant. We must have $a_{11}>\max \left\{a_{00}, a_{01}\right\}>a_{10}$. Let $r_{i}(x)=r$ for some constant $r \in\left(\max \left\{a_{00}, a_{01}\right\}, a_{11}\right)$ for all $x \in X$ and all $i \in N$.

The expected utilities for strategies 0 and 1 are, respectively, given by

$$
\begin{aligned}
& u_{0}(x, r)=(1-x)\left(a_{00}-r-B\right)+x\left(a_{01}-r-B\right), \\
& u_{1}(x, r)=(1-x)\left(a_{10}-r-B\right)+x\left(a_{11}-r\right) .
\end{aligned}
$$

For sufficiently large $B$, strategy 1 is the unique best response for $x \in[1 / 2,1]$. Since $u_{0}(x, r)$ and $u_{1}(x, r)$ are continuous on $x \in(0,1)$, this proves that all-1 is uniquely stochastically stable. The claim follows.

Lemma 3.2 and 3.3 both utilize the fact that choosing the payoff dominant strategy helps reference-dependent agents to reduce losses. Next, we provide the first main result of the paper:

Theorem 3.4. (I) If all-s is uniquely stochastically stable under the prospect dynamic with BRM choice rule for all models of reference points and all forms of the value function satisfying conditions (i)-(v) of Assumption 2.1, then $s$ is super-dominant.

(II) If $s$ is super-dominant, then all-s is stochastically stable under the prospect dynamic with BRM choice rule for all models of reference points and all forms of the value function satisfying condition (iv) of Assumption 2.1. Furthermore, all-s is uniquely stochastically stable if s is super-dominant and the condition below holds:

$$
\liminf _{x \searrow \frac{1}{2}} u_{0}\left(x, r_{i}(x)\right)-u_{1}\left(x, r_{i}(x)\right)>0,
$$

\footnotetext{
${ }^{11}$ Note that Theorem 6.1 in Sawa and Wu (2018) shows that loss-dominance is sufficient for stochastic stability only if $\beta$ is not sufficiently small.
} 
for any $i \in N$ and any $n>\underline{n}$, for some $\underline{n}>0$.

Proof. Proof of claim (I):

We follow Sawa and Wu (2018) to prove the necessity of the first condition of Definition 3.1. Without loss of generality, suppose that strategy 0 does not satisfy the first condition of Definition 3.1, which implies that $a_{01}<a_{10}$. Assume that agents have preferences given by Eq.(1) with $\alpha=\beta=1$, i.e. loss averse preferences with no diminishing sensitivity, and have a constant reference point $r$ that satisfies $a_{01}<r<\min \left\{a_{10}, a_{11}\right\}$. Then, the all-0 state is not stochastically stable for sufficiently large $\lambda$. This is because for all $x \in[0,1]$ and any value of $a_{00}$, if $\lambda$ is sufficiently large,

$$
u_{0}(x, r)=(1-x) v\left(a_{00}, r\right)+x \lambda\left(a_{01}-r\right)<u_{1}(x, r)=(1-x)\left(a_{10}-r\right)+x\left(a_{11}-r\right) .
$$

For the second condition of Definition 3.1, Lemma 3.2 and 3.3 show that if $s$ fails to satisfy the second condition, then there exist reference-dependent preferences under which all-s is not stochastically stable.

Finally, we consider the case in which both conditions of Definition 3.1 hold weakly. In this case, we have $a_{11}=a_{00}$ and $a_{10}=a_{01}$. Symmetry in payoffs immediately implies that both all-0 and all-1 are stochastically stable. Hence, neither of them are uniquely stochastically stable. To conclude, if all- $s$ is uniquely stochastically stable for all reference-dependent models, then $s$ is super-dominant.

Proof of claim (II):

Without loss of generality, let 0 be the super-dominant strategy, which implies that $a_{00} \geq a_{11}>$ $a_{01} \geq a_{10}$, with at least one of the two weak inequalities being strict. Hence, by condition (iv) of Assumption 2.1, we have $v\left(a_{00}, r_{i}(x)\right) \geq v\left(a_{11}, r_{i}(x)\right)$ and $v\left(a_{01}, r_{i}(x)\right) \geq v\left(a_{10}, r_{i}(x)\right)$ with at least one weak inequality being strict for any $r_{i}(x)$. Then we have, for any $x \in[0,1 / 2]$, any $r_{i}(x)$ and any $v(\cdot)$ that satisfies condition (iv) of Assumption 2.1,

$$
\begin{aligned}
u_{0}\left(x, r_{i}(x)\right) & =(1-x) v\left(a_{00}, r_{i}(x)\right)+x v\left(a_{01}, r_{i}(x)\right) \\
& >(1-x) v\left(a_{11}, r_{i}(x)\right)+x v\left(a_{10}, r_{i}(x)\right) \\
& \geq x v\left(a_{11}, r_{i}(x)\right)+(1-x) v\left(a_{10}, r_{i}(x)\right)=u_{1}\left(x, r_{i}(x)\right) .
\end{aligned}
$$

Thus, 0 is the unique best response for all $x \in[0,1 / 2]$. This proves the first statement, i.e., all-0 is stochastically stable. Note that for even $n$, the basin of attraction of all- 0 includes $\left\{0, \ldots, \frac{1}{2}\right\}$, while the basin of attraction of all-1 is a subset of $\left\{\frac{n-2}{2 n}, \ldots, 1\right\}$. The former is strictly larger than the latter. Thus, if $n$ is even, all-0 is uniquely stochastically stable.

Next, we prove the second statement. If condition (9) holds, then 0 is the unique best response of all agents in state $x=\frac{n+1}{2 n}$ for all sufficiently large $n$. This implies that the basin of attraction 
of all-0 includes $\left\{0, \ldots, \frac{n+1}{2 n}\right\}$ for all sufficiently large odd $n$, while the basin of attraction of all- 1 is a subset of $\left\{\frac{n+3}{2 n}, \ldots, 1\right\}$.

Note that when all agents adopt the same constant reference point $r \in \mathbb{R}$, strategy 0 being super-dominant together with condition (iv) of Assumption 2.1 implies that $v\left(a_{00}, r\right)+v\left(a_{01}, r\right) \geq$ $v\left(a_{11}, r\right)+v\left(a_{10}, r\right)$. This is the condition for 0 being the risk-dominant strategy in the transformed payoff matrix shown in Table 2 .

Table 2: $2 \times 2$ Coordination Game (Value Function)

Player 2

\begin{tabular}{|c|c|c|c|}
\hline \multirow{3}{*}{ Player 1} & & 0 & 1 \\
\hline & 0 & $v\left(a_{00}, r\right), v\left(a_{00}, r\right)$ & $v\left(a_{01}, r\right), v\left(a_{10}, r\right)$ \\
\hline & 1 & $v\left(a_{10}, r\right), v\left(a_{01}, r\right)$ & $v\left(a_{11}, r\right), v\left(a_{11}, r\right)$ \\
\hline
\end{tabular}

Hence, the same logic of equilibrium selection in Kandori et al. (1993) and Young (1993) applies to our setting and all-0 is stochastically stable.

Theorem 3.4 provides a positive result in regard to super-dominance. It shows that a superdominant equilibrium is the unique social convention selected by agents with reference-dependent preferences because it helps agents to avoid not only risks but also losses.

As shown in Theorem 3.4, condition (iv) of Assumption 2.1 is the key condition for stochastic stability. In addition, claim (I) of the theorem concerns the other conditions of the assumption. When a strategy $s$ fails to be super-dominant, some reference-dependent preferences satisfying all conditions (i)-(v) of Assumption 2.1 exist such that all- $s$ is not stochastically stable. Condition (9) in claim (II) is satisfied by a broad class of preferences including all continuous preferences, i.e. continuous value functions with continuous reference point functions, and some discontinuous preferences such as the loss-avoidance preferences in Example 2. We hope that the result will provide guidance for future experimental investigation of equilibrium selection problem in games played by subjects with reference-dependent preferences.

\subsection{The logit choice rule and instability of super-dominance}

Although we find that super-dominant equilibrium is uniquely stochastically stable under the prospect dynamic with BRM choice rule, the second main result of the paper demonstrates that it is no longer the case if the agents instead employ the logit choice rule. The difference in re- 
sults between the two choice rules is caused by the fact that stochastic stability for the logit choice rule is characterized by states that maximize the potential function of the underlying game, i.e. $\operatorname{argmax}_{x \in X} \sum_{0 \leq x^{\prime} \leq x} u_{1}\left(x^{\prime}\right)-u_{0}\left(x^{\prime}\right)$. However, a state that maximizes the potential does not necessarily have a greater basin of attraction when the payoff difference function is nonlinear.

Theorem 3.5. Suppose that $s$ is super-dominant and $s^{\prime} \neq s$. There exists a value function $v(\cdot)$ satisfying conditions (i)-(v) of Assumption 2.1 such that all-s' ${ }^{\prime}$ is uniquely stochastically stable under the prospect dynamic with logit choice rule.

Proof. Without loss of generality, assume that 0 is super-dominant, i.e., $a_{00} \geq a_{11}>a_{01} \geq a_{10}$. Let

$$
\bar{x}=\frac{a_{00}-a_{10}}{a_{00}-a_{01}+a_{11}-a_{10}} .
$$

At state $\bar{x}$, the expected monetary outcomes of the two strategies are the same. Strategy 0 being risk-dominant implies that $\bar{x}>\frac{1}{2}$. Assume that all agents have the following reference point:

$$
r(x)= \begin{cases}a_{10} & \text { for } x \in[0, \bar{x}], \\ a_{11} & \text { for } x \in(\bar{x}, 1],\end{cases}
$$

which is state-dependent. Also, all agents have loss-avoidance preferences given by Eq.(2). In this case, 0 is unique best response for $x<\bar{x}$ and 1 is so for $x>\bar{x}$.

We prove the claim by showing that $\sum_{x \in X} u_{1}(x)-u_{0}(x)>0$. Observe that $u_{0}(x)-u_{1}(x) \leq$ $a_{00}-a_{10}$ for $x \leq \bar{x}$, and that $u_{1}(x)-u_{0}(x)>(2 x-1) B$ for $x>\bar{x}$. Hence, we have

$$
\begin{aligned}
& \sum_{x \leq \bar{x}} u_{0}(x)-u_{1}(x)<n \bar{x}\left(a_{00}-a_{10}\right), \\
& \sum_{x>\bar{x}} u_{1}(x)-u_{0}(x)>n\left(1-\bar{x}-\frac{1}{n}\right)(2 \bar{x}-1) B .
\end{aligned}
$$

For sufficiently large $B>0$, we have that

$$
\begin{aligned}
\sum_{x \in X} u_{1}(x)-u_{0}(x) & =\sum_{x \leq \bar{x}} u_{1}(x)-u_{0}(x)+\sum_{x>\bar{x}} u_{1}(x)-u_{0}(x) \\
& >n\left(1-\bar{x}-\frac{1}{n}\right)(2 \bar{x}-1) B-n \bar{x}\left(a_{00}-a_{10}\right)>0 .
\end{aligned}
$$

This implies that all-1, or the super-dominated equilibrium, is uniquely stochastically stable.

The intuition behind Theorem 3.5 is as follows. The severity of suboptimal choices, i.e. payoff deficits, is an important determinant of stochastic stability under logit choice rule because the 
unlikeliness of transitions is captured by payoff deficits faced by agents who revise their strategies. When $B$ is sufficiently large for agents with loss-avoidance preferences, payoff deficits associated with strategy 0 in population state $x \in(\bar{x}, 1]$, where strategy 1 is the unique best response, would be sufficiently large to make all-0 unstable.

The next example illustrates that when agents have loss-averse preferences with diminishing sensitivity, a super-dominated equilibrium can be uniquely stochastically stable under the prospect dynamic with logit choice rule.

Example 5 (Loss aversion). Consider a game with monetary payoffs $a_{00}=2, a_{01}=a_{10}=0$ and $a_{11}=1$. Assume that all agents have state-dependent reference point given as follows: ${ }^{12}$

$$
r(x)= \begin{cases}0 & \text { for } x \in[0,2 / 3], \\ 1 & \text { for } x \in(2 / 3,1]\end{cases}
$$

Agents have loss-averse preferences given by Eq.(1) with $\alpha=\beta \in(0,1]$ and $\lambda \geq 1$. Let $\bar{x}=2 / 3$, which corresponds to the mixed-strategy equilibrium of the game. Observe that $u_{0}(x)-u_{1}(x)=$ $2^{\alpha}(1-x)-x<2^{\alpha}$ for $x \leq \bar{x}$, and that $u_{1}(x)-u_{0}(x)=\lambda(2 x-1)-(1-x)>\lambda(2 \bar{x}-1)+\bar{x}-1$ for $x>\bar{x}$. Hence, we have

$$
\begin{aligned}
& \sum_{x \leq \bar{x}} u_{0}(x)-u_{1}(x)<2^{\alpha} n \bar{x}=\frac{2^{\alpha+1} n}{3}, \quad \sum_{x>\bar{x}} u_{1}(x)-u_{0}(x)>\frac{n}{3}\left(\frac{1}{3}-\frac{1}{n}\right)(\lambda-1), \\
& \sum_{x>\bar{x}} u_{1}(x)-u_{0}(x)>\sum_{x \leq \bar{x}} u_{0}(x)-u_{1}(x) \quad \Rightarrow \quad \lambda>\frac{3 \cdot 2^{\alpha+1} n}{n-3}+1 .
\end{aligned}
$$

All-1 is stochastically stable under the prospect dynamic with logit choice rule if the degree of loss aversion $\lambda$ is sufficiently large. The condition will be reduced to $\lambda>3 \cdot 2^{\alpha+1}+1$ as the population size $n$ approaches infinity.

The intuition behind Example 5 is similar to that of Theorem 3.5. Strong loss-aversion creates large payoff deficits associated with the super-dominant strategy, which makes the super-dominant equilibrium unstable.

Although we show that super-dominant equilibrium is not stochastically stable given some reference-dependent preferences under the prospect dynamic with logit choice rule. The following proposition provides a positive result on super-dominant equilibrium given that agents adopt a common constant reference point.

\footnotetext{
${ }^{12}$ Note that we use the same reference point as in the proof of Theorem 3.5.
} 
Proposition 3.6. Suppose that the value function adopted by the agents satisfies condition (iv) of Assumption 2.1 and all agents adopt the same constant reference point $r \in \mathbb{R}$. Then all-s is uniquely stochastically stable under the prospect dynamic with logit choice rule for all forms of the value function if and only if $s$ is super-dominant.

Proof of Proposition 3.6. 'if' part:

Without loss of generality, let strategy 0 be the super-dominant strategy, which implies that $a_{00} \geq$ $a_{11}>a_{01} \geq a_{10}$, with at least one of the two weak inequalities being strict. Hence, by condition (iv) of Assumption 2.1, $v\left(a_{00}, r\right) \geq v\left(a_{11}, r\right)$ and $v\left(a_{01}, r\right) \geq v\left(a_{10}, r\right)$ with at least one weak inequality being strict. Then we have

$$
\begin{aligned}
\sum_{x \in X} u_{1}(x)-u_{0}(x) & =\sum_{x \in X}(1-x)\left(v\left(a_{10}, r\right)-v\left(a_{00}, r\right)\right)+\sum_{x \in X} x\left(v\left(a_{11}, r\right)-v\left(a_{01}, r\right)\right) \\
& =\frac{n+1}{2}\left(v\left(a_{11}, r\right)+v\left(a_{10}, r\right)-v\left(a_{00}, r\right)-v\left(a_{01}, r\right)\right)<0 .
\end{aligned}
$$

Therefore, all-0 is uniquely stochastically stable.

'only if' part:

We will show that if strategy 0 fails to be super-dominant, there exists some reference-dependent preference for which all-1 is stochastically stable.

Case 1: Suppose strategy 0 is payoff-dominant but fails to be the maximin strategy. We must have $a_{00} \geq a_{11} \geq a_{10}>a_{01}$. Assume that agents have loss-averse preferences given by Eq.(1) with no diminishing sensitivity (i.e. $\alpha=\beta=1$ ) and $r \in\left(a_{01}, a_{10}\right)$. We have

$$
\begin{aligned}
\sum_{x \in X} u_{1}(x)-u_{0}(x) & =\frac{n+1}{2}\left(v\left(a_{11}, r\right)+v\left(a_{10}, r\right)-v\left(a_{00}, r\right)-v\left(a_{01}, r\right)\right) \\
& =\frac{n+1}{2}\left(\left(a_{11}-r\right)+\left(a_{10}-r\right)-\left(a_{00}-r\right)-\lambda\left(a_{01}-r\right)\right) .
\end{aligned}
$$

When $\lambda$ is sufficiently large, the above expression is strictly positive, implying that all-1 is uniquely stochastically stable.

Case 2: Suppose strategy 0 is the maximin strategy but not payoff-dominant. The proof of this case is similar to the proof of Lemma 3.2. Consider loss-averse preferences given by Eq.(1) and let $r$ be such that $a_{11}>r>\max \left\{a_{00}, a_{01}\right\}$. Choose $\delta \in(0,1)$, and there exists a sufficiently small $\beta<1$ such that

$$
\begin{aligned}
\sum_{x \in X} u_{1}(x)-u_{0}(x) & =\frac{n+1}{2}\left(\left(a_{11}-r\right)^{\alpha}-\lambda\left(r-a_{10}\right)^{\beta}+\lambda\left(r-a_{00}\right)^{\beta}+\lambda\left(r-a_{01}\right)^{\beta}\right) \\
& >\frac{n+1}{2}\left(\left(a_{11}-r\right)^{\alpha}+\lambda \delta\right)>0 .
\end{aligned}
$$


Hence, all-1 is uniquely stochastically stable.

Case 3: Suppose strategy 0 is neither payoff-dominant nor the maximin strategy, then strategy 1 must be the super-dominant strategy. By the proof of the 'if' part, we have that all-1 is uniquely stochastically stable.

Remark 3.7. When the reference point function is constant, the payoff difference between the two strategies, $u_{1}(x)-u_{0}(x)$, is linear in $x$. From Eq.(10) in the proof of Proposition 3.6, one can observe that

$$
\operatorname{sgn}\left[\sum_{x \in X} u_{1}(x)-u_{0}(x)\right]=\operatorname{sgn}\left[v\left(a_{11}, r\right)+v\left(a_{10}, r\right)-v\left(a_{00}, r\right)-v\left(a_{01}, r\right)\right],
$$

where $\operatorname{sgn}[a]=1$ if $a>0, \operatorname{sgn}[a]=0$ if $a=0$, and $\operatorname{sgn}[a]=-1$ otherwise. This observation together with our discussion in Section 3.2 implies that the sets of stochastically stable states coincide among the BRM rule and the logit choice if the reference point function is constant.

Remark 3.8. The proof of Case 1 in the 'only if' part shows, as a byproduct, that a strategy being a maximin strategy is a necessary condition for stochastic stability even if we restrict preference models to the ones with no diminishing sensitivity, e.g. $\alpha=\beta=1$ in Eq.(1). This observation leads to a counterpart of the only if part of Theorem 3.2, which is a result under the BRM rule, in Sawa and $\mathrm{Wu}$ (2018). That is, all-s is uniquely stochastically stable under the logit choice rule for all models of reference points and all forms of the value function with no diminishing sensitivity only if $s$ is risk-dominant and a maximin strategy.

\section{Discussion}

\subsection{The tightness for Assumption 2.1}

We have shown that given any preferences satisfying condition (iv) of Assumption 2.1, a superdominant equilibrium, if exists, is uniquely stochastically stable. In this section, we show that a super-dominated equilibrium can be stochastically stable if condition $(i v)$ is violated.

Proposition 4.1. If $v(\cdot, \cdot)$ fails to satisfy (iv) of Assumption 2.1, then there exists some game for which a super-dominated equilibrium is stochastically stable under some reference-dependent preferences.

Proof. Assume that all agents have a value function $v(\cdot)$ and adopt an exogenous reference point equal to $r$. Suppose for some $a^{*}>a^{* *}, v\left(a^{*}, r\right)=v\left(a^{* *}, r\right)$ for some $r \in \mathbb{R}$. Let $a_{00}=a^{*}$, 
$a_{01}=a_{10}<a^{*}$ and $a_{11}=a^{* *}$. Given this payoff structure, strategy 0 is the super-dominant strategy, and we have $v\left(a_{00}, r\right)=v\left(a_{11}, r\right)$ and $v\left(a_{01}, r\right)=v\left(a_{10}, r\right)$. This implies that both all-0 and all-1, which is super-dominated, are stochastically stable.

\subsection{Ordinal potential function and loss evaluation function}

In this section, we hope to provide some further explanations for our results. We define an ordinal potential function for the coordination games under each strategy choice rule, and relate it to stochastic stability. We further define a function, which we call a loss evaluation function, that maps a state to the difference in losses associated with the two strategies. The loss evaluation function enables us to obtain graphic illustrations for the difference between the BRM and the logit choice rule.

To simplify the analysis in this section, we restrict preferences to be loss-averse given by Eq.(1), and we assume that the common reference point function $r:[0,1] \rightarrow \mathbb{R}$ is Riemann integrable on $[0,1] .{ }^{13}$ Define ordinal potential functions (Monderer and Shapley, 1996) for the BRM rule and the logit rule as follows.

$$
\begin{aligned}
& I_{B R M}(x ; \lambda)=\int_{0}^{x} \operatorname{sgn}_{+}\left[u_{1}(y)-u_{0}(y)\right]-\operatorname{sgn}_{+}\left[u_{0}(y)-u_{1}(y)\right] d y, \\
& I_{\text {logit }}(x ; \lambda)=\int_{0}^{x} u_{1}(y)-u_{0}(y) d y,
\end{aligned}
$$

where $\operatorname{sgn}_{+}[a]=1$ if $a>0$, and $\operatorname{sgn}_{+}[a]=0$ otherwise. Note that the potential of all- 0 under each choice rule is zero, i.e. $I_{B R M}(0 ; \lambda)=I_{\text {logit }}(0 ; \lambda)=0$, and that both potential functions have local maximum points at $x=0$ and $x=1$. The next result states that the state maximizing the potential under a given choice rule is stochastically stable.

Proposition 4.2. If $I_{C h}(1 ; \lambda)<0$, then all-0 is uniquely stochastically stable under the choice rule Ch. If $I_{C h}(1 ; \lambda)>0$, then all-1 is uniquely stochastically stable under the choice rule Ch.

The proposition resembles the results proved by studies that employ a Riemann-integral argument to determine stochastically stabile states, e.g. Blume (2003); Maruta (2002); Sandholm (2010a). The proof is straightforward. For example, $I_{B R M}(1 ; \lambda)<0$ implies that the basin of attraction of all-0 is strictly greater than that of all-1 in the limit of large population size. Hence, all-0 is uniquely stochastically stable. We omit the formal proof.

\footnotetext{
${ }^{13}$ All reference point functions in our examples have at most finite discontinuities, i.e. Riemann integrable.
} 
Let function $L:[0,1] \times \mathbb{R} \rightarrow \mathbb{R}$ be such that

$$
L(x, a)= \begin{cases}(r(x)-a)^{\beta} & \text { if } a<r(x) \\ 0, & \text { otherwise }\end{cases}
$$

where $r(x)$ is the common reference point function. $L(x, a)$ gives the loss perceived by an agent who receives monetary payoff $a$ in state $x$. Define the loss-evaluation function $L: X \rightarrow \mathbb{R}$ as

$$
L(x)=(1-x)\left(L\left(x, a_{00}\right)-L\left(x, a_{10}\right)\right)+x\left(L\left(x, a_{01}\right)-L\left(x, a_{11}\right)\right) .
$$

When an agent chooses strategy $i$, the perceived loss associated with the strategy is $(1-x) L\left(x, a_{i 0}\right)+$ $x L\left(x, a_{i 1}\right)$ multiplied by $\lambda . L(x)$ gives the difference in the perceived losses of the two strategies for $\lambda=1$. The next result relates $L(x)$ to the maximizers of the ordinal potential function for each choice rule. ${ }^{14}$

Proposition 4.3. Suppose that preferences are given by Eq.(1).

(i) If $\int_{0}^{1} \operatorname{sgn}_{+}[-L(x)] d x>1 / 2$, then $I_{B R M}(1 ; \lambda)<0$ for all sufficiently large $\lambda$. If $\int_{0}^{1} \operatorname{sgn}_{+}[L(x)] d x>$ $1 / 2$, then $I_{B R M}(1 ; \lambda)>0$ for all sufficiently large $\lambda$.

(ii) If $\int_{0}^{1} L(x) d x<0$, then $I_{\text {logit }}(1 ; \lambda)<0$ for all sufficiently large $\lambda$. If $\int_{0}^{1} L(x) d x>0$, then $I_{\text {logit }}(1 ; \lambda)>0$ for all sufficiently large $\lambda$.

Proof. Let $G(x, a)=(a-r(x))^{\alpha}$ if $a \geq r(x)$, and $G(x, a)=0$ otherwise. Define

$$
G(x)=(1-x)\left(G\left(x, a_{00}\right)-G\left(x, a_{10}\right)\right)+x\left(G\left(x, a_{01}\right)-G\left(x, a_{11}\right)\right),
$$

Observe that the utility difference can be written as $u_{0}(x)-u_{1}(x)=G(x)-\lambda L(x)$, and that $L(x)$ and $G(x)$ do not depend on $\lambda$.

Proof of claim (i):

If $L(x)<0$, then $u_{0}(x)-u_{1}(x)>0$ for all sufficiently large $\lambda$. This implies that for all sufficiently large $\lambda$, if $\frac{|\{x \in X: L(x)<0\}|}{n}>\frac{1}{2}$, then $\sum_{x \in X} \operatorname{sgn}_{+}\left[u_{1}(x)-u_{0}(x)\right]-\operatorname{sgn}_{+}\left[u_{0}(x)-u_{1}(x)\right]<0$; and if $\frac{|\{x \in X: L(x)<0\}|}{n}<\frac{1}{2}$, then $\sum_{x \in X} \operatorname{sgn}_{+}\left[u_{1}(x)-u_{0}(x)\right]-\operatorname{sgn}_{+}\left[u_{0}(x)-u_{1}(x)\right]>0 . \quad \frac{|\{x \in X: L(x)<0\}|}{n}$ is approximated by the integral $\int_{0}^{1} \operatorname{sgn}_{+}[-L(x)] d x$ and the summation $\frac{1}{n} \sum_{x \in X} \operatorname{sgn}_{+}\left[u_{1}(x)-u_{0}(x)\right]-$ $\operatorname{sgn}_{+}\left[u_{0}(x)-u_{1}(x)\right]$ is approximated by $I_{B R M}(1 ; \lambda)$ in the limit of large population size.

Proof of claim (ii):

Observe that $\sum_{x \in X} u_{0}(x)-u_{1}(x)=\sum_{x \in X} G(x)-\lambda \sum_{x \in X} L(x)$. This implies that if $\sum_{x \in X} L(x)<$

\footnotetext{
${ }^{14}$ A similar function is defined in Sawa and $\mathrm{Wu}$ (2018), which shows an analogous result to (i) in Proposition 4.3.
} 
0 , then $\sum_{x \in X} u_{1}(x)-u_{0}(x)<0$, and if $\sum_{x \in X} L(x)>0$, then $\sum_{x \in X} u_{1}(x)-u_{0}(x)>0$ for all sufficiently large $\lambda$. The summation $\frac{1}{n} \sum_{x \in X} L(x)$ is approximated by the integral $\int_{0}^{1} L(x) d x$ and the summation $\frac{1}{n} \sum_{x \in X} u_{1}(x)-u_{0}(x)$ is approximated by $I_{\text {logit }}(1 ; \lambda)$ in the large population limit.

The intuition for Proposition 4.3 is as follows. When $\lambda$ is sufficiently large, the difference in perceived losses dominates the difference in perceived gains, implying that a strategy associated with a lower loss becomes the unique best response. Hence, we can use the loss evaluation function to determine stochastically stable states under the BRM choice rule. Furthermore, the utility difference between the two strategies increases as $\lambda$ becomes larger. Hence, for sufficiently large $\lambda$, we can use the integration of the loss evaluation function to determine stochastically stable states under the logit choicer rule.

As Theorem 3.5 suggests, the potential maximizers (stochastically stable states) for the BRM rule and those for the logit choice do not necessarily coincide. ${ }^{15}$ The next example provides a graphic illustration for how one can use the loss evaluation function to determine the potential maximizers under the BRM and the logit choice rules.

Example 6. Consider a game with $a_{00}=10, a_{01}=1, a_{10}=0$ and $a_{11}=9$. Strategy 0 is superdominant. Assume that all agents have the preferences given by Eq.(1) with $\alpha=\beta=1$, and the state-dependent reference point given by $r(x)=1$ for $x \in[0,2 / 3]$, and $r(x)=9$ for $x \in(2 / 3,1]$. The loss evaluation function is depicted by the kinked line in Figure 1. For comparison, the straight line in Figure 1 shows the loss evaluation function for a constant reference point, $r=5$.

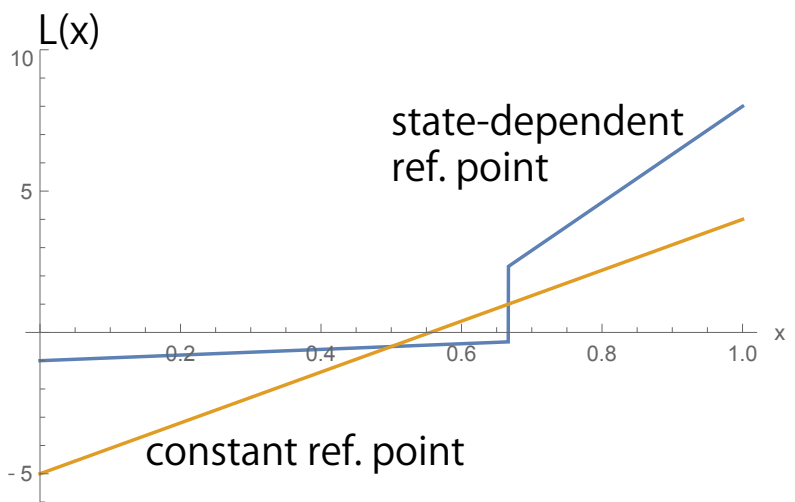

Figure 1: The loss evaluation function

\footnotetext{
${ }^{15}$ In the special case that the reference point is constant, they do coincide (Proposition 3.6).
} 
The point where each line crosses the $\mathrm{x}$-axis is greater than $x>.5$. Proposition 4.3 implies that all-0 is the unique potential maximizer for large $\lambda$ under the BRM rule, which is consistent with Theorem 3.4.

For the logit choice rule, $\int_{0}^{1} L(x) d x>0$ holds for the state-dependent model. Hence, all-1 is the unique potential maximizer for large $\lambda$. On the other hand, $\int_{0}^{1} L(x) d x<0$ holds for the constant reference point. Hence, all-0 is the unique potential maximizer for large $\lambda$, which is consistent with Proposition 3.6.

\subsection{Matching without Self-Matching}

We consider a matching protocol without self-matching and discuss the robustness of our results. For every agent $i$, define

$$
z_{i}(x)= \begin{cases}\frac{n x}{n-1} & \text { if agent } i \text { plays strategy } 0 \\ \frac{n x-1}{n-1} & \text { if agent } i \text { plays strategy } 1\end{cases}
$$

That is, $z_{i}(x)$ is the fraction of agents playing strategy 1 over all agents except agent $i$. When agent $i$ is not matched with herself, her utility of playing strategy $s$ and her reference point depend on the strategy distribution of the other $n-1$ agents, i.e., $z_{i}(x)$. Agent $i$ 's utility is written as

$$
u_{s}\left(z_{i}(x), r_{i}\left(z_{i}(x)\right)\right)=\left(1-z_{i}(x)\right) v\left(a_{s 0}, r_{i}\left(z_{i}(x)\right)\right)+z_{i}(x) v\left(a_{s 1}, r_{i}\left(z_{i}(x)\right)\right) .
$$

The next proposition generalizes our results to settings with the matching protocol without self-matching.

Proposition 4.4. For all sufficiently large n, Theorem 3.4 and Proposition 3.6 are valid for settings with the matching protocol without self-matching.

Proof. For the 'only if' part, the counter-examples in the proofs of Theorem 3.4 and Proposition 3.6 also work for the matching without self-matching. The proof of the 'if' part of Proposition 3.6 is straightforward. We prove the 'if' part of Theorem 3.4 for this matching protocol.

Let strategy 0 be the super-dominant strategy, i.e. $a_{00} \geq a_{11}>a_{01} \geq a_{10}$, with at least one of the two weak inequalities being strict. Hence, $v\left(a_{00}, r_{i}\left(z_{i}(x)\right)\right) \geq v\left(a_{11}, r_{i}\left(z_{i}(x)\right)\right)$ and $v\left(a_{01}, r_{i}\left(z_{i}(x)\right)\right) \geq$ $v\left(a_{10}, r_{i}\left(z_{i}(x)\right)\right)$ with at least one weak inequality being strict for all $i \in N$. For any $x \in[0,1 / 2]$ and any $v(\cdot)$ that satisfies Assumption 2.1, we have that for all sufficiently large $n$,

$$
u_{0}\left(z_{i}(x), r_{i}\left(z_{i}(x)\right)\right)=\left(1-z_{i}(x)\right) v\left(a_{00}, r_{i}\left(z_{i}(x)\right)\right)+z_{i}(x) v\left(a_{01}, r_{i}\left(z_{i}(x)\right)\right)
$$




$$
\begin{aligned}
& >\left(1-z_{i}(x)+\frac{1}{n-1}\right) v\left(a_{11}, r_{i}\left(z_{i}(x)\right)\right)+\left(z_{i}(x)-\frac{1}{n-1}\right) v\left(a_{10}, r_{i}\left(z_{i}(x)\right)\right) \\
& \geq z_{i}(x) v\left(a_{11}, r_{i}\left(z_{i}(x)\right)\right)+\left(1-z_{i}(x)\right) v\left(a_{10}, r_{i}\left(z_{i}(x)\right)\right)=u_{1}\left(z_{i}(x), r_{i}\left(z_{i}(x)\right)\right) .
\end{aligned}
$$

The strict inequality is implied by the assumption that 0 is super-dominant and the fact that $1 /(n-1)$ is close to zero for sufficiently large $n$. For the weak inequality, observe that for all $x \in[0,1 / 2]$ and all $i \in N$,

$$
z_{i}(x) \leq \frac{n x}{n-1} \quad \Rightarrow \quad 1-z_{i}(x)+\frac{1}{n-1} \geq z_{i}(x)
$$

Then $a_{11}>a_{10}$ implies the weak inequality. Therefore, strategy 0 is the unique best response for all agents if $x \in[0,1 / 2]$, which implies that all-0 is uniquely stochastically stable.

\section{Conclusion}

This paper incorporates insights from behavioral economics into the study of game theory. We investigate what social conventions would emerge in $2 \times 2$ coordination games through evolutionary dynamics in a population of agents with reference-dependent preferences.

We define a concept called super-dominance which combines three important concepts in game theory: risk-dominance, payoff-dominance and the maximin strategy. In $2 \times 2$ coordination games, the super-dominant strategy (if exists) is arguably the most salient strategy that agents would coordinate on. We first find that a population state in which all agents adopt the same strategy is uniquely stochastically stable under dynamic with BRM choice rule if and only if the strategy is super-dominant. We call this population state the super-dominant equilibrium. The result demonstrates that the super-dominant equilibrium constitutes a robust social convention that will be selected by agents with reference-dependent preferences in the long run if agents adopt the BRM choice rule. However, we also find that super-dominance does not guarantee stochastic stability under dynamic with the logit choice rule. For any $2 \times 2$ coordination game with a super-dominant strategy, there exists a model of reference-dependent preferences with which the super-dominant equilibrium fails to be stochastically stable.

We hope that this study will encourage further investigations on how psychological biases influence the long run behavior of individuals in a society. 


\section{Acknowledgments}

The authors thank the Editor and three anonymous reviewers for helpful comments and constructive

feedback on the paper. Sawa acknowledges financial support from JSPS Grant-in-Aid 15K17023 and 18K12740.

\section{References}

Battalio, R., Samuelson, L., and Huyck, J. V. (2001). Optimization incentives and coordination failure in laboratory stag hunt games. Econometrica, 69:749-764.

Bergin, J. and Lipman, B. L. (1996). Evolution with state-dependent mutations. Econometrica, 64:943-956.

Bilancini, E. and Boncinelli, L. (2016). The evolution of conventions under condition-dependent mistakes. Unpublished Manuscript, Università degli Studi di Firenze.

Blume, L. (1993). The statistical mechanics of strategic interaction. Games and Economic Behavior, $5: 387-424$.

Blume, L. (2003). How noise matters. Games and Economic Behavior, 44:251-271.

Cachon, G. P. and Camerer, C. F. (1996). Loss-avoidance and forward induction in experimental coordination games. Quarterly Journal of Economics, 111:165-194.

Cournot, A. A. (1838). Researches into the Mathematical Principles of the Theory of Wealth. Macmillan, New York. Translated by N. T. Bacon (1897).

Dokumacı, E. and Sandholm, W. H. (2011). Large deviations and multinomial probit choice. Journal of Economic Theory, 146:2151-2158.

Durrett, R. (2005). Probability: Theory and Examples. Duxbury Press, third edition.

Ellison, G. (2000). Basins of attraction, long-run stochastic stability, and the speed of step-by-step evolution. Review of Economic Studies, 67:17-45.

Feltovich, N. (2011). The effect of subtracting a constant from all payoffs in a hawk-dove game: Experimental evidence of loss aversion in strategic behavior. Southern Economic Journal, 77:814826. 
Feltovich, N., Iwasaki, A., and Oda, S. H. (2012). Payoff levels, loss avoidance, and equilibrium selection in games with multiple equilibria: An experimental study. Economic Inquiry, 50:932952.

Foster, D. P. and Young, H. P. (1990). Stochastic evolutionary game dynamics. Theoretical Population Biology, 38:219-232.

Freidlin, M. I. and Wentzell, A. D. (1998). Random perturbations of dynamical systems. Springer Verlag, New York, second edition.

Harsanyi, J. C. and Selten, R. (1988). A General Theory of Equilibrium Selection in Games. MIT Press, first edition.

Hwang, S.-H., Lim, W., Neary, P., and Newton, J. (2018). Conventional contracts, intentional behavior and logit choice: Equality without symmetry. Games and Economic Behavior, 110:273294.

Hwang, S.-H. and Newton, J. (2017). Payoff-dependent dynamics and coordination games. Economic Theory, 64:589-604.

Hwang, S.-H. and Rey-Bellet, L. (2017). Positive feedback in coordination games: stochastic evolutionary dynamics and the logit choice rule. arXiv. arXiv:1701.04870v3 [cs.GT].

Kahneman, D. (2011). Thinking, fast and slow. Farrar, Straus and Giroux, first edition.

Kahneman, D. and Tversky, A. (1979). Prospect theory: An analysis of decision under risk. Econometrica, 47:263-292.

Kandori, M., Mailath, G. J., and Rob, R. (1993). Learning, mutation, and long run equilibria in games. Econometrica, 61:1003-1037.

Klaus, B. and Newton, J. (2016). Stochastic stability in assignment problems. Journal of Mathematical Economics, 62:62-74.

Knetsch, J. L. and Sinden, J. A. (1984). Willingness to pay and compensation demanded: Experimental evidence of an unexpected disparity in measures of value. Quarterly Journal of Economics, 99:507-521.

Köszegi, B. and Rabin, M. (2006). A model of reference-dependent preferences. Quarterly Journal of Economics, 121:1133-1165. 
Kreindler, G. E. and Young, H. P. (2013). Fast convergence in evolutionary equilibrium selection. Games and Economic Behavior, 80:39-67.

Kreindler, G. E. and Young, H. P. (2014). Rapid innovation diffusion in social networks. Proceedings of the National Academy of Sciences, 111:10881-10888.

Lim, W. and Neary, P. R. (2016). An experimental investigation of stochastic adjustment dynamics. Games and Economic Behavior, 100:208-219.

Maruta, T. (2002). Binary games with state dependent stochastic choice. Journal of Economic Theory, 103:351-376.

Mäs, M. and Nax, H. H. (2016). A behavioral study of "noise" in coordination games. Journal of Economic Theory, 162:195-208.

Monderer, D. and Shapley, L. S. (1996). Potential games. Games and Economic Behavior, 14:124$-143$.

Morris, S. (2000). Contagion. Review of Economic Studies, 67:57-78.

Myatt, D. P. and Wallace, C. C. (2003). A multinomial probit model of stochastic evolution. Journal of Economic Theory, 113:286-301.

Naidu, S., Hwang, S.-H., and Bowles, S. (2010). Evolutionary bargaining with intentional idiosyncratic play. Economics Letters, 109(1):31-33.

Neary, P. R. (2012). Competing conventions. Games and Economic Behavior, 76(1):301-328.

Newton, J. (2018a). The deconstruction of conventions. Unpublished Manuscript, Available at SSRN: https://ssrn.com/abstract $=3216269$.

Newton, J. (2018b). Evolutionary game theory: A renaissance. Games, 9(2):31.

Newton, J. and Angus, S. D. (2015). Coalitions, tipping points and the speed of evolution. Journal of Economic Theory, 157:172-187.

Newton, J. and Sawa, R. (2015). A one-shot deviation principle for stability in matching problems. Journal of Economic Theory, 157:1—-27.

Peski, M. (2010). Generalized risk-dominance and asymmetric dynamics. Journal of Economic Theory, 145(1):216-248. 
Rydval, O. and Ortmann, A. (2005). Loss avoidance as selection principle: Evidence from simple stag-hunt games. Economics Letters, 88:101-107.

Sandholm, W. H. (2010a). Orders of limits for stationary distributions, stochastic dominance, and stochastic stability. Theoretical Economics, 5:1-26.

Sandholm, W. H. (2010b). Population Games and Evolutionary Dynamics. MIT Press, first edition.

Sandholm, W. H. and Staudigl, M. (2016). Large deviations and stochastic stability in the small noise double limit. Theoretical Economics, 11:279-355.

Sawa, R. and Wu, J. (2018). Prospect dynamic and loss dominance. Unpublished Manuscript, Available at SSRN: http://dx.doi.org/10.2139/ssrn.2804852.

Schmidt, D., Shupp, R., Walker, J. M., and Ostrom, E. (2003). Playing safe in coordination games: the roles of risk dominance, payoff dominance, and history of play. Games and Economic Behavior, 42:281-299.

Staudigl, M. (2012). Stochastic stability in asymmetric binary choice coordination games. Games and Economic Behavior, 75:372-401.

Staudigl, M. and Weidenholzer, S. (2014). Constrained interactions and social coordination. Journal of Economic Theory, 152:41-63.

Thaler, R. (1980). Toward a positive theory of consumer choice. Journal of Economic Behavior and Organization, 1:39-60.

Tversky, A. and Kahneman, D. (1992a). Advances in prospect theory: Cumulative representation of uncertainty. Journal of Risk and Uncertainty, 5:297-323.

Tversky, A. and Kahneman, D. (1992b). Advances in prospect theory: Cumulative representation of uncertainty. Journal of Risk and Uncertainty, 5:297-323.

von Neumann, J. and Morgenstern, O. (1944). Theory of Games and Economic Behavior. Princeton University Press, first edition.

Young, H. P. (1993). The evolution of conventions. Econometrica, 61:57-84.

Young, H. P. (2011). The dynamics of social innovation. Proceedings of the National Academy of Sciences, 108:21285-21291. 